\title{
Disruption of Poly(ADP-ribose) Polymerase (PARP) Protects Against Stress-Evoked Immunocompromise
}

\author{
Deborah L. Drazen, ${ }^{1}$ Donna Bilu, ${ }^{1}$ Nancy Edwards, ${ }^{1}$ and Randy J. Nelson ${ }^{2}$ \\ ${ }^{1}$ Department of Psychology, The Johns Hopkins University, Baltimore, MD USA \\ ${ }^{2}$ Departments of Psychology and Neuroscience, The Ohio State University, Columbus OH USA \\ Accepted September 27, 2001
}

\begin{abstract}
Background: Chronic stress, mediated by adrenal hormones, is a major risk factor in the progression and outcome of human disease. While the secretion of adrenal hormones is known to be the primary endocrine mediator of stress-induced immunocompromise, the molecular mechanisms underlying the immunocompromise remain unspecified. Overproduction of the nuclear enzyme, poly (ADP-ribose) polymerase (PARP) has been implicated in the molecular pathway that leads to cell death by energy depletion following stress.

Materials and Methods: Wild-type (WT) mice and mice with targeted disruption of the gene encoding PARP-1 (PARP-1 -/-) were subjected to 2 wk daily cold-water swim; splenocyte proliferation, anti-KLH IgG, and serum corticosterone concentrations were assessed. Additional mice of each genotype received daily i.p. injections of dexamethasone (DEX) $(0.75 \mathrm{mg} / \mathrm{kg})$ for $2 \mathrm{wk}$, and splenocyte proliferation and anti-KLH IgG were assessed.
\end{abstract}

Results: Splenocyte proliferation and specific antibody concentrations of stressed WT mice were reduced by $\sim 20 \%$ of their pre-stress levels. In contrast, PARP-1 $-/-$ mice maintained normal cell-mediated and humoral immune function following enforced cold-water swim stress. PARP-1 $-/-$ mice also failed to compromise immune function following DEX treatment, whereas WT mice displayed significant reductions of immune function following this treatment.

Conclusions: These results provide support for the involvement of PARP activation in immunological damage following physical stress. These results suggest that glucocorticoid-induced immunosuppression may require the activation of PARP in order for apoptosis of immune cells to take place. Taken together, these results suggest that therapies designed to inhibit PARP may prove valuable in the treatment of stress-related diseases.

\section{Introduction}

Stress is a major determinant of disease onset and progression in humans (1-3), and it also contributes significantly to disease processes among domesticated and wild animals (4-6). One of the many physiological complications associated with chronic stress is compromised immune function. It is well documented that psychological and physical stressors impair humoral and cell-mediated immunity $(7,8)$. The ability to cope successfully with stressors influences susceptibility to disease, the severity of symptoms during disease, and ultimately morbidity caused by disease. For example, stressors can influence the progression and outcome of several human diseases including cancer, AIDS, cardiac, respiratory, and autoimmune diseases $(7,9-11)$. Stressors as diverse as cold-water swim, immobilization, forced running, unpredictable electric shock, scholastic exam preparation, or health care provision for chronically-ill relatives have all been reported to reduce lymphocyte numbers and function in human and non-human animals (12-14).

Address correspondence and reprint requests to: Randy J. Nelson, Departments of Psychology and Neuroscience, The Ohio State University, Columbus, OH, 43210, USA. Phone: (614) 538-9526; fax: (614) 451-3116; e-mail: rnelson@osu.edu.
Indeed, it is well known that both psychological and physical stressors modulate the hypothalamicpituitary-adrenal (HPA) axis, leading to increased glucocorticoid release which, in turn, modulates immune function in both human and non-human animals (2). For example, caring for chronically-ill patients is known to create persistent stress in caregivers, and this in turn has adverse health consequences. Caregivers of the elderly experience higher psychological distress and have higher salivary cortisol concentrations than non-caregivers, and this in turn may directly decrease mitogen-induced lymphocyte proliferation (12); caregivers of age-matched dementia patients also have reduced cellular immune responses (15). Other stressors, such as long-term exercise and strenuous work, reduce lymphocyte numbers and this reduction is correlated with high circulating cortisol concentrations (16).

While numerous experiments have implicated elevated glucocorticoid activity as a mediator of stress-induced physical problems, little is known of the specific cellular or molecular mechanisms involved in the process. One possible molecular mechanism mediating the effects of stress on immune function involves stress-induced activation of glucocorticoid receptors and the subsequent inhibition of specific gene products directly on immune cells (17). 
Perhaps the most extensively documented immunosuppressive effect of glucocorticoids involves peripheral $\mathrm{T}$ cells. Glucocorticoids can affect the development of $\mathrm{T}$ helper (Th) cells (favoring the generation of Th2 cells). Glucocorticoids also downregulate FasL expression, and thus inhibit $\mathrm{T}$ cell apoptosis. Under other circumstances, glucocorticoids are potent inducers of apoptosis; glucocorticoid concentrations achieved during a typical stress response can kill $\mathrm{CD} 4(+)$ and $\mathrm{CD} 8(+)$ thymocytes $(17,18)$.

One potential pathway through which stress and, subsequently, glucocorticoids influence apoptosis induction in immune cells is via activation of the nuclear enzyme, poly (ADP-ribose) polymerase (PARP). Excessive DNA damage leads to increased PARP activity; PARP, in turn, catalyzes attachment of ADP ribose units from NAD to nuclear proteins $(19,20)$. Excessive activation of PARP can deplete NAD and ATP; ATP is consumed during the regeneration of NAD, leading to cell death by energy depletion. PARP appears to be involved in protection against a wide range of physiological insults. For example, mice lacking the gene for PARP are resistant to the development of experimentallyinduced cerebral ischemia (21), and are protected from experimentally-induced Parkinson's disease (22), heart attacks (23), and streptozotocin-induced diabetes (24). The range of physiological complications associated with chronic stress is similar to that in which deletion of PARP plays a protective role, including cerebral ischemia, heart attack, and diabetes (25-27). As discussed above, stress also influences many aspects of immune function. Therefore, it is likely that inhibition of PARP may also play a protective role against the damaging effects of stress on immune function.

In order to assess the role of PARP in mediating stress-induced immunocompromise, mice genetically engineered to lack the PARP-1 gene (PARP-1 $-/-$ ) were subjected to daily, cold-water swim and both humoral and cell-mediated immune parameters were assessed. To test the possibility of glucocorticoid resistance in PARP-1 - /- mice, animals were treated with dexamethasone (DEX) and immune function was assessed. We show here that targeted disruption of the gene that encodes for PARP-1 protects against stress-induced immunocompromise.

\section{Materials and Methods}

Animals

Mice were generously provided by Drs. Ted and Valina Dawson of The Johns Hopkins School of Medicine. Once in our laboratory, animals were individually housed in polycarbonate cages $(28 \times$ $17 \times 12 \mathrm{~cm})$ in colony rooms with a 16:8-h lightdark (LD) cycle [lights on at $0700 \mathrm{~h}$ Eastern Standard Time] and were allowed to acclimate for $2 \mathrm{wk}$ prior to the onset of the experiment. Colony rooms were maintained with an ambient temperature of $21 \pm 2{ }^{\circ} \mathrm{C}$, and relative humidity was held constant at $50 \pm 5 \%$. Food (LabDiet 5001; PMI Nutrition, Brentwood, MO) and tap water were provided ad libitum throughout the course of the study.

Twenty-four male mice that were genetically engineered to lack the PARP-1 gene (PARP-1 - - ), and 24 male animals that were wild-type (WT) control mice of the same background strain (129 SvEv; 28) were used in the present study. Twelve additional animals of each genotype were handled daily, but not subjected to the cold-water swim. In all other ways, they were treated as the experimental mice.

\section{Experimental Procedures}

Experiment 1. Animals were acclimated to the lab for 2 weeks prior to experimentation. A baseline blood sample was then taken from all mice for later assessment of baseline corticosterone values. Throughout the study blood sampling procedure was kept to less than 2 minutes for each animal, disallowing sufficient time for serum coticosterone concentrations to increase significantly during the procedure. Furthermore, all animals in all groups experienced the same blood sampling procedure. After 1 additional week, mice were individually subjected to 14 consecutive days of 3-min forced cold-water swims (after which they were towel-dried and returned to their individual cages). After 4 days of forced swim, animals received a single subcutaneous injection of $100 \mu \mathrm{g}$ of the antigen keyhole limpet hemocyanin (KLH), to which the animals were previously naive, suspended in $0.1 \mathrm{ml}$ sterile saline (Day 0 ) and were then returned to the colony room. KLH is an innocuous respiratory protein derived from the giant keyhole limpet (Megathura crenulata). KLH was used because it generates a robust antigenic response in rodents, but does not make the animals ill (e.g., cause inflammation or fever) (29). Blood (500 $\mu \mathrm{l})$ was drawn from the retro-orbital sinus 10 days post-immunization. This sampling period was chosen in order to capture peak IgG production during the course of the immune response to KLH (30). On each sampling day, animals were brought into the surgery room individually and lightly anesthetized with methoxyflurane vapors (Metofane, Medical Developments, Melbourne, Australia), and blood samples were obtained between $1000 \mathrm{~h}$ and $1200 \mathrm{~h}$ EST. Samples were allowed to clot for $l \mathrm{~h}$, the clots were removed, and the samples centrifuged (at $4^{\circ} \mathrm{C}$ ) for $30 \mathrm{~min}$ at $2500 \mathrm{rpm}$. Serum aliquots were aspirated and stored in sealable polypropylene microcentrifuge tubes at $-80^{\circ} \mathrm{C}$ until assayed for anti-KLH IgG, and serum corticosterone concentrations. On the last day of sampling (Day 10) animals were killed by cervical dislocation, and spleens were removed under aseptic conditions, 
suspended in culture medium (RPMI), and used immediately to assess splenocyte proliferation.

Experiment 2. To evaluate more directly the possibility of glucucorticoid resistance in mice lacking the gene for PARP-1, additional WT and PARP-1 - / - mice $(n=10 / g e n o t y p e)$ were subjected to DEX treatment. Animals were injected daily with DEX $(0.75 \mathrm{mg} /$ $\mathrm{kg}$, i.p.) for $2 \mathrm{wk}$. This regime produces robust immunosuppression mice (31). KLH was injected after 4 days of DEX treatment, and blood samples were taken 10 days later for anti-KLH IgG. Animals were killed and spleens were removed for splenocyte proliferation as described for Experiment 1.

\section{Humoral Immunity}

To assess humoral immunity, serum anti-KLH IgG concentrations were assayed using an enzyme-linked immunosorbant assay (ELISA). Microtiter plates were coated with antigen by incubating overnight at $4^{\circ} \mathrm{C}$ with $0.5 \mathrm{mg} / \mathrm{ml} \mathrm{KLH}$ in sodium bicarbonate buffer ( $\mathrm{pH}=9.6)$, washing with phosphate-buffered saline (PBS; $\mathrm{pH}=7.4$ ) containing $0.05 \%$ Tween 20 (PBS-T; $\mathrm{pH}=7.4$ ), then blocking with $5 \%$ nonfat dry milk in PBS-T overnight at $4^{\circ} \mathrm{C}$ to reduce nonspecific binding, and finally washing again with PBS-T. Thawed serum samples were diluted 1:100 with PBS-T, and $300 \mu \mathrm{l}$ of each serum dilution was added in duplicate to the wells of the antigen-coated plates. Positive control samples (pooled sera from mice previously determined to have high levels of anti-KLH IgG antibody, similarly diluted with PBS-T) and negative control samples (pooled sera from KLH-naive mice, similarly diluted with PBS-T) were also added in duplicate to each plate. Plates were sealed, incubated at $37^{\circ} \mathrm{C}$ for $3 \mathrm{~h}$, then washed with PBS-T. Secondary antibody (alkaline phosphatase-conjugated-anti-mouse IgG diluted 1:1000 with PBS-T; Cappel, Costa Mesa, CA) was added to the wells, and the plates were sealed and incubated for $1 \mathrm{~h}$ at $37^{\circ} \mathrm{C}$. Plates were washed again with PBS$\mathrm{T}$ and $150 \mu \mathrm{l}$ of the enzyme substrate p-nitrophenyl phosphate (Sigma Chemical, St, Louis, MO: $1 \mathrm{mg} / \mathrm{ml}$ in diethanolamine substrate buffer) was added to each well. Plates were protected from light during the enzyme-substrate reaction, which was terminated after 20 min by adding $50 \mu \mathrm{l}$ of $1.5 \mathrm{M} \mathrm{NaOH}$ to each well. The optical density (OD) of each well was determined using a plate reader (Bio-Rad: Benchmark, Richmond, CA) equipped with a $405 \mathrm{~nm}$ wavelength filter, and the mean OD for each set of duplicate wells was calculated. To minimize intra-assay variability, the mean OD for each sample was expressed as a percent of its plate positive control OD for statistical analyses.

\section{Cell-Mediated Immunity}

Cell-mediated immunity was assessed by measuring splenocyte proliferation, using a colorimetric assay based on the tetrazolium salt 3-(4,5demethylthiazol-2-yl)-5-(3-carboxymethoxyphenyl)-
2-(4-sulfophenyl)-2H-tetrazolium (MTS). Splenocytes were separated from tissue by compressing the whole spleen between sterile frosted glass slides; separated cells were suspended in $3 \mathrm{ml}$ of culture medium (RPMI-1640/Hepes supplemented with $1 \%$ penicillin $(5000 \mathrm{U} / \mathrm{ml}) /$ streptomycin $(5000 \mu \mathrm{l} / \mathrm{ml})$, $1 \%$ L-glutamine $(2 \mathrm{mM} / \mathrm{ml}), 0.1 \%$ 2-mercaptoethanol $\left(5 \times 10^{-2} \mathrm{M} / \mathrm{ml}\right)$, and $10 \%$ heat-inactivated fetal bovine serum). Splenocyte counts and viability were determined with a hemacytometer and trypan blue exclusion. Viable cells (which exceed $95 \%$ ) were adjusted to $2 \times 10^{6}$ cells $/ \mathrm{ml}$ by dilution with culture medium, and $50 \mu \mathrm{l}$ aliquots of each cell suspension (i.e., 100,000 cells) were added to the wells of sterile flat-bottom 96-well culture plates. Con A (Sigma Chemical Co., St. Louis, MO) which specifically stimulates $\mathrm{T}$ cells to proliferate, was diluted with culture medium to concentrations of $40,20,10$, and $0 \mu \mathrm{g} / \mathrm{ml}$. It was determined that the optimal dose of Con A was $20 \mu \mathrm{g} / \mathrm{ml} .50 \mu \mathrm{l}$ of each mitogen concentration and $50 \mu \mathrm{l}$ of culture medium were added to the wells of the plate that would contain the spleen cell suspensions to yield a final volume of $100 \mu \mathrm{l} / \mathrm{well}$ (each in duplicate). Cell suspensions were added to the plates last. Plates were incubated at $37^{\circ} \mathrm{C}$ with $5 \% \mathrm{CO}_{2}$ for $48 \mathrm{~h}$ prior to addition of $20 \mu \mathrm{l}$ of MTS/PMS solution (Promega; $0.92 \mathrm{mg} / \mathrm{ml}$ of phenazine methosulphate (PMS) in sterile Dulbecco's phosphate buffered saline) per well. Plates were then incubated at $37^{\circ} \mathrm{C}$ with $5 \% \mathrm{CO}_{2}$ for an additional $4 \mathrm{~h}$. The OD of each well was determined with a microplate reader (BioRad: Benchmark) equipped with a $490 \mathrm{~nm}$ wavelength filter. Mean OD values for each set of duplicates were used in subsequent statistical analyses.

\section{Corticosterone Radioimmunoassay}

Serum corticosterone concentrations were determined by radioimmunoassay (RIA) using the ICN Biomedicals (Costa Mesa, CA) ${ }^{125}$ I double-antibody kit. The corticosterone assay was highly specific, cross-reacting at less than $0.3 \%$ with other steroid hormones, according to manufacturer's specifications. Serum corticosterone values were determined in a single RIA. The intra-assay coefficients of variation were all $<10 \%$.

\section{Statistical Analyses}

Anti-KLH IgG, splenocyte proliferation, and serum corticosterone data were analyzed using a series of 2 (genotype) $\times 2$ (swim test or not) analyses of variance (ANOVA). The DEX data were analyzed similarly using a series of 2 (genotype) $\times 2$ (drug treatment) ANOVAs. Post-hoc comparisons for significant main effects were evaluated using the Tukey-HSD test. Group differences were considered statistically significant when $\mathrm{p}<0.05$.

\section{Results}

Experiment 1. As has been observed many times (32), WT mice that were forced to swim had lower levels of 
anti-KLH IgG in their serum on Day 14 of swimming than WT mice not forced to swim $(p<0.05)$ (Fig. 1A). In contrast, anti-KLH IgG was not changed by forced daily swimming in PARP-1 $-/-$ mice relative to PARP-1 $-/-$ mice that were not forced to swim $(p>0.05)$. Pre-stress anti-KLH IgG did not differ between WT and PARP-1 $-/-$ mice $(p>0.05)$ (Fig. 1A). Raw absorbances at $405 \mathrm{~nm}$ are WT prestressed $134.7+4.9$, WT stressed $104.4+5.3$, PARP-1 $-/-$ pre-stressed $139.6+5.1$ and PARP-1 -/stressed $143.1+5.7$.

Splenocyte proliferation was reduced in WT mice subjected to cold water swim compared with WT mice not subjected to cold water swim $(p<0.05)$ (Fig. 1B). In PARP-1 -/- mice, however, splenoctye proliferation was not altered by forced cold-water swim, compared with PARP-1 -/- mice not subjected to swim $(p>0.05)$. Pre-stress splenocyte proliferation did not differ between WT and PARP-1 - /mice $(p>0.05)$ (Fig. 1B).

In WT mice, cold-water swim elevated serum corticosterone concentrations by approximately $20 \%$ compared to WT mice not forced to swim $(\mathrm{p}<0.05)$ (Fig. 1C). In PARP-1 -/- mice, cold water swim increased corticosterone concentrations (approximately $35 \%$ ) compared with PARP-1 - / - mice not forced to swim $(\mathrm{p}<0.05)$. Basal corticosterone concentrations did not differ between WT and PARP-1 -/- mice $(\mathrm{p}>0.05)$ (Fig. 1C)

Experiment 2. After 2 weeks of DEX treatment, both anti-KLH IgG concentrations and splenocyte proliferation were reduced in WT mice $(p<0.05$ in both cases) (Figs. 2A and B). In PARP-1 $-/-$ mice, however, 2 weeks of DEX treatment had no effect on either anti-KLH IgG or splenoctye proliferation ( $p>0.05$ in both cases) (Figs. 2A and B). Raw absorbances at $405 \mathrm{~nm}$ are WT $65.8+16.9$ and PARP-1 - / - $101.9+18.6$.

\section{Discussion}

Chronic or acute stress, whether physical, psychological or somatic, can have dramatic effects on physiology generally, and on immune function specifically. The mechanisms by which stressors and the consequent increased output of the HPA axis affect immune function have been a major focus within the field of psychoneuroimmunology. The present results identify a pronounced protection from stress-induced immunosuppression in PARP-1 -/- mice. Specifically, both humoral and cell-mediated immune parameters were significantly reduced (compromised) in WT mice which were forced to swim in cold water once a day for two weeks. Similar observations have been reported by other groups (32-34). This is consistent with the hypothesis that certain stressors cause physiological changes that render animals more susceptible to disease processes. In contrast, mice with a targeted deletion of PARP-1 had normal basal levels of immune parameters assessed, and
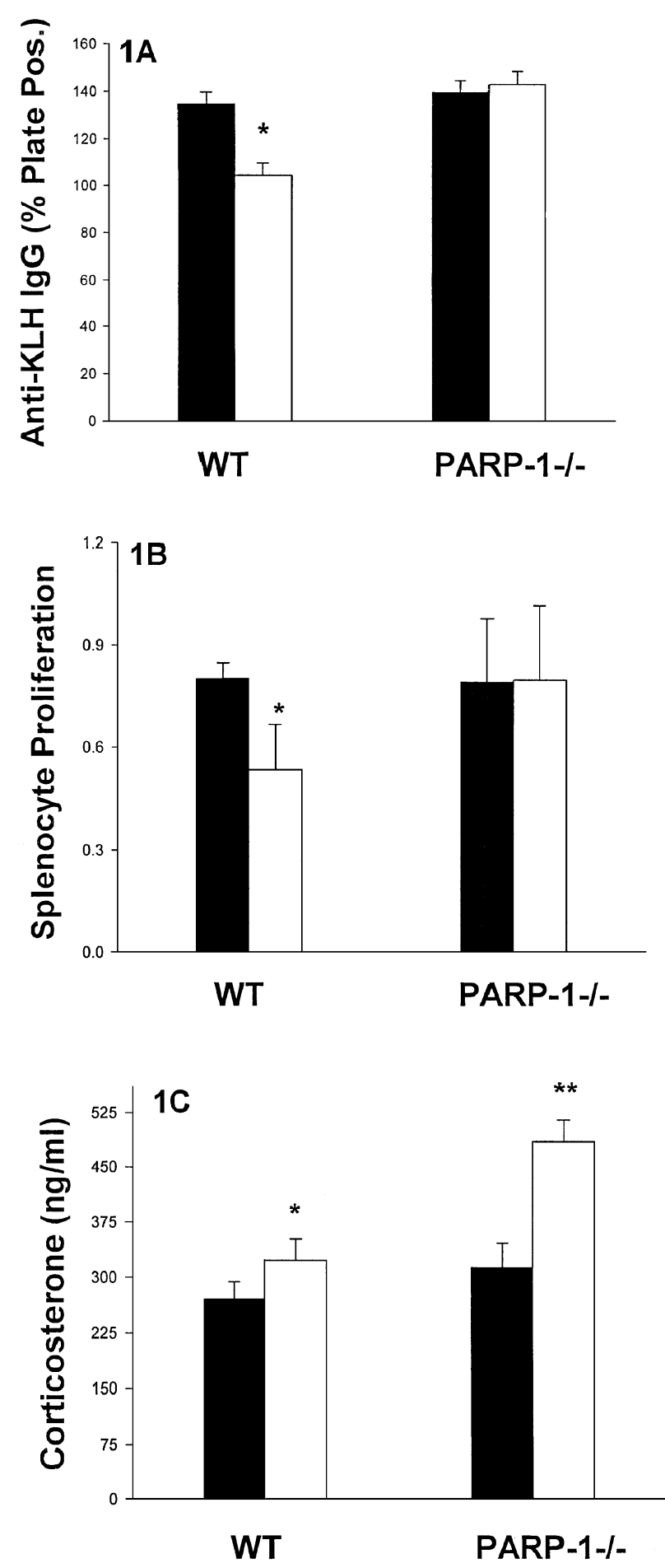

Fig. 1. Experiment 1. In all cases, closed bars represent baseline values, and open bars represent values obtained after 2 wk swim stress. (A) Mean ( \pm SEM) serum anti-KLH immunoglobulin G concentrations (represented as a percentage of the plate positive). (B) Mean ( \pm SEM) splenocyte proliferation values (represented as absorbance units). (C) Mean ( \pm SEM) serum corticosterone concentrations $(\mathrm{ng} / \mathrm{ml})$. Closed bars represent baseline, pre-stress values, and open bars represent values obtained after 2 wk stress. * Significantly greater than WT, baseline; ** Significantly greater than all other groups $(p<0.05)$. 

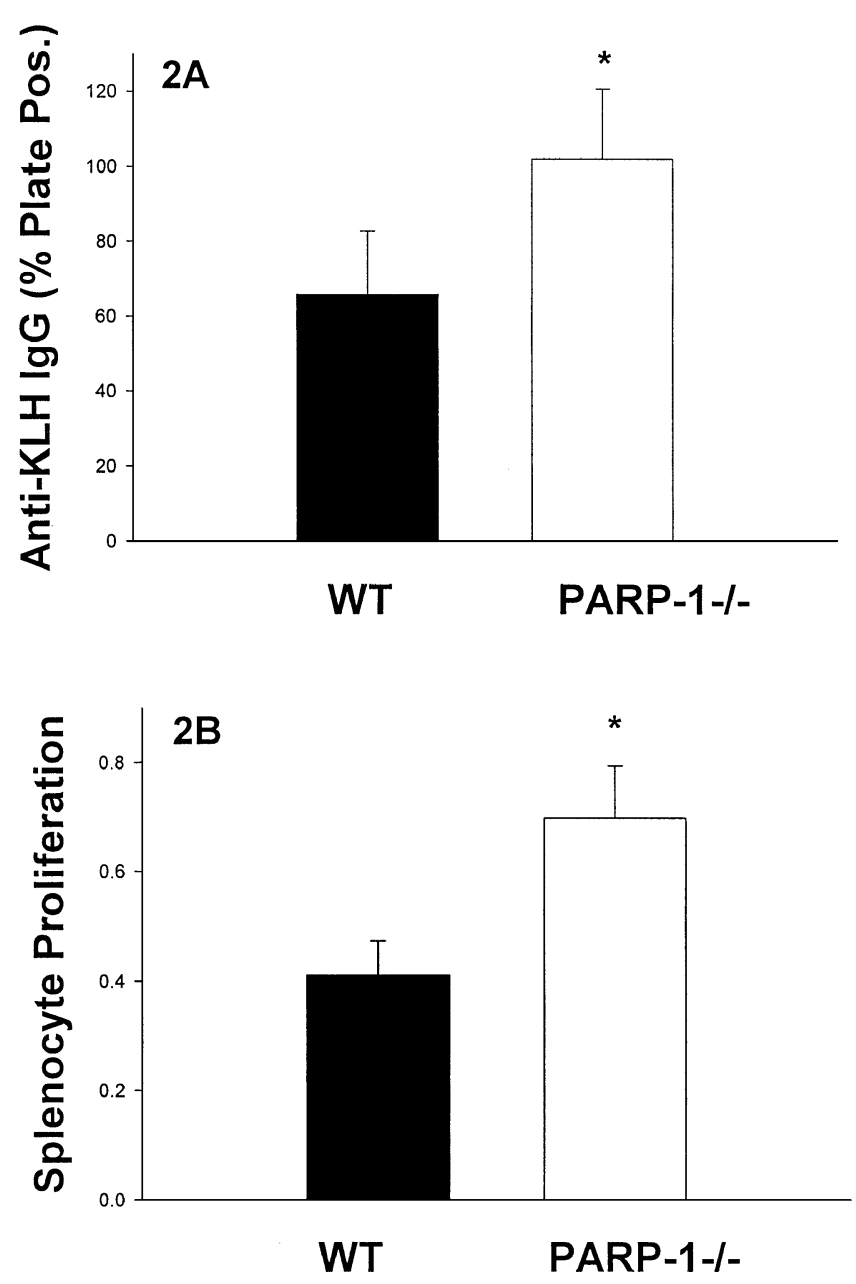

Fig. 2. Experiment 2. (A) Mean $( \pm$ SEM) serum anti-KLH immunoglobulin $\mathrm{G}$ concentrations (represented as percentage of plate positive) after $2 \mathrm{wk}$ dexamethasone treatment. (B) Mean $( \pm \mathrm{SEM})$ splenocyte proliferation values (represented as absorbance units) after $2 \mathrm{wk}$ dexamethasone treatment. * Significantly greater than WT $(p<0.05)$.

these parameters were not compromised in cohorts subjected to daily swim.

The observation that PARP-1 -/- mice had an increase in corticosterone when stressed, as did WT mice, suggests that PARP intervenes downstream of glucocorticoid action to compromise immune function. That is, the immunoprotection observed in PARP-1 $-/-$ mice is not due to a reduced response to stress in these animals, at least as defined by secretion of glucocorticoids. In fact, WT mice subjected to cold-water swim had a $20 \%$ increase in corticosterone whereas PARP-1 $-/-$ mice had a $35 \%$ increase in corticosterone in the same situation. These data imply that the protection observed in PARP-1 $-/-$ mice is not the result of reduced HPA activity. The fact that elevated glucocorticoids caused immunocompromise in WT but not the knockout mice in Experiment 2 further implies that PARP-1 -/mice have reduced responsiveness to the effects of glucocorticoids.
The present results are consistent with previous studies in which targeted deletion of the PARP-1 gene provided protection from an array of physiological insults $(21,22,24)$. In the absence of PARP-1, therefore, it appears that both endogenous and exogenous glucocorticoids are unable to inhibit cellmediated and humoral immune function. These results suggest indirectly that glucocorticoid-induced immunocompromise may require the activation of PARP in order to induce apoptosis in immune cells. PARP-1, therefore, may be a crucial link between stress and immune function.

While the present results make a compelling case for the involvement of PARP in mediating the effects of glucocorticoids on the immune system, it must be recognized that the present findings share the same limitations as others using knockout animals. Mice with targeted disruption of genes may use compensatory mechanisms that preserve the functions of the protein derived from the disrupted gene because gene deletion occurs in an early embryonic stage (35). It is also possible that compensation by other enzymes capable of poly ADPribosylation (VPARP, PARP-2; 36,37) may be partially responsible for the effects observed in PARP-1 -/mice in the present study. Studies using specific pharmacological inhibitors of PARP will be needed to confirm the present conclusions, when available.

Taken together, these results provide convincing evidence for a principal involvement of PARP activation in immunological damage following stress. Mice lacking the gene for PARP-1 are resistant to the immunocompromising effects of glucocorticoids. Further, this is true whether the glucocorticoids are endogenously secreted in response to a stressor, or administered exogenously. Therefore, our results suggest that PARP plays a crucial role in glucocorticoid-mediated immunocompromise. Future studies must clarify the duration of the benefits provided in the absence of PARP. Therapies designed towards inhibiting PARP may improve the treatment of stressrelated diseases that are linked to compromised immunity.

\section{Acknowledgments}

The authors thank Brian Spar for technical support and Dr. Stephen Woods for helpful comments on the manuscript. We are also very grateful to Drs. Ted Dawson and Valina Dawson of The Johns Hopkins University School of Medicine for generously providing us with the experimental animals used this study. In addition, we thank Kim Nies for expert animal care. Supported by NIH grants MH 57760 (RJN) and NSF grant IBN 00-0080745.

\section{References}

1. Balbin EG, Ironson GH, Solomon GF. (1999) Stress and coping: the psychoneuroimmunology of HIV/AIDS. Baillieres Best Pract. Res. Clin. Ednocrinol. Metab. 13: 615-633. 
2. Chrousos GP. (2000) Stress, chronic inflammation, and emotional and physical well-being: concurrent effects and chronic sequelae. J. Allergy Clin. Immunol. 106: 275-291.

3. Yang EV, Glaser R. (2000) Stress-induced immunomodulation: impact on immune defenses against infectious disease. Biomed. Pharmacother. 54: 245-250.

4. Swain MG, Patchev V, Vergalla J, et al. (1993) Suppression of hypothalamic-pituitary-adrenal axis responsiveness to stress in a rat model of acute cholestasis. J. Clin. Invest. 91: 1903-1908.

5. Malan FS, Horak IG, de Vos V, van Wyk JA. (1997) Wildlife parasites: lessons for parasite control in livestock. Vet. Parasitol. 71: 137-153.

6. Kraabel BJ, Miller MW. (1997) Effect of simulated stress on susceptibility of bighorn sheep neutrophils to Pasteurella haemolytica leukotoxin. J. Wildl. Dis. 33: 558-566.

7. McEwen BS, Seeman T. (1999) Protective and damaging effects of mediators of stress. Elaborating and testing the concepts of allostasis and allostatic load. Ann. N.Y. Acad. Sci. USA 896: 30-47.

8. Birmaher B, Rabin BS, Garcia MR, et al. (1994) Cellular immunity in depressed, conduct disorder, and normal adolescents: role of adverse life events. J. Am. Acad. Child Adolesc. Psychiatry 33: 671-678.

9. Strange KS, Kerr LR, Andrews HN, et al. (2000) Psychosocial stressors and mammary tumor growth: an animal model. Neurotoxicol. Teratol. 22: 89-102.

10. Elenkov IJ, Chrousos GP. (1999) Stress, cytokine patterns and susceptibility to disease. Baillieres Best Pract. Res. Clin. Endocrinol. Metab. 13: 583-595.

11. Kiecolt-Glaser JK, Glaser R. (1999) Psychoneuroimmunology and cancer: fact or fiction? Eur. J. Cancer 35: 1603-1607.

12. Bauer ME, Vedhara K, Perks P, et al. (2000) Chronic stress in caregivers of dementia patients is associated with reduced lymphocyte sensitivity to glucocorticoids. J. Neuroimmunol. 103: 84-92.

13. Lacey K, Zaharia MD, Griffiths J, et al. (2000) A prospective study of neuroendocrine and immune alterations associated with the stress of an oral academic examination among graduate students. Psychoneuroendocrinol. 25: 339-356.

14. Cohen S, Miller GE, Rabin BS. (2001) Psychological stress and antibody response to immunization: a critical review of the human literature. Psychosom. Med. 63: 7-18.

15. Cacioppo JT, Poehlmann KM, Kiecolt-Glaser JK, et al. (1998) Cellular immune responses to acute stress in female caregivers of dementia patients and matched controls. Health Psychol. 17: 182-189.

16. Weicker H, Werle E. (1991) Interaction between hormones and the immune system. Int. J. Sports Med. 12: S30-S37.

17. Ashwell JD, Lu FW, Vacchio MS. (2000) Glucocorticoids in T cell development and function. Ann. Rev. Immunol. 18: 309-345.

18. Rook GA. (1999) Glucocorticoids and immune function. Baillieres Best Pract. Res. Clin. Endocrinol. Metab. 13: 567-581.

19. Nosseri C, Coppola S, Ghibelli L. (1994) Possible involvement of Poly(ADP-Ribosyl) polymerase in triggering stressinduced apoptosis. Exp. Cell Res. 212: 367-373.

20. Pieper AA, Verma A, Zhang J, Snyder S. (1999) Poly (ADPribose) polymerase, nitric oxide, and cell death. Trends Pharmacol. Sci. 20: 171-181.
21. Eliasson MJL, Sampei K, Mandir AS, et al. (1997) Poly (ADP-ribose) polymerase gene Disruption renders mice resistant to cerebral ischemia. Nature Med. 3: 1089-1095.

22. Mandir AS, Przedborski S, Jackson-Lewis V, et al. (1999) Poly (ADP-ribose) polymerase activation mediates 1-methyl-4phenyl-1,2,3,6-tetrahydropyridine (MPTP)-induced parkinsonism. Proc. Natl. Acad. Sci. USA 96: 5774-5779.

23. Pieper AA, Walles T, Wei G, et al. (2000) Myocardial postischemic injury is reduced by poly ADP ribose polymerase-1 gene disruption. Mol. Med. 6: 271-282.

24. Pieper AA, Brat DJ, Krug DK, et al. (1999) Poly (ADP-ribose) polymerase-deficient mice are protected from streptozotocininduced diabetes. Proc. Natl. Acad. Sci. 96: 3059-3064.

25. Cossette S, Frasure-Smith N, Lesperance F. (2001) Clinical implications of a reduction in psychological distress on cardiac prognosis in patients participating in a psychosocial intervention program. Psychosom. Med. 63: 257-266.

26. Haines A, Cooper J, Meade TW. (2001) Psychological characteristics and fatal ischaemic heart disease. Heart 85: 385389.

27. Naliboff BD, Goldstein IB, Shapiro D, Frank HJ. (1988) Mental and physical stress as moderators of the postural response in insulin-dependent diabetic patients. Health. Psychol. 7: 499-514.

28. Wang ZQ, Stingl L, Morrison C, et al. (1997) PARP is important for genomic stability but dispensable in apoptosis. Genes Dev. 11: 2347-2358.

29. Dixon F, Jacot-Guillarmod H, McConahey PJ. (1966) The antibody responses of rabbits and rats to hemocyanin. $J$. Immunol. 97: 350-355.

30. Drazen DL, Kriegsfeld LJ, Schneider JE, Nelson RJ. (2000) Leptin, but not immune function, is linked to reproductive responsiveness. Am. J. Physiol. 278: 1401-1407.

31. Tsutsui N, Kamiyama T. (1998) Suppression of in vitro IFNgamma production by spleen cells of Plasmodium chabaudiinfected C57BL/10 mice exposed to dexamethasone at a low dose. Int. J. Immunopharmacol. 20: 141-152.

32. DeVries AC, Gerber JM, Richardson HN, et al. (1997) Stress affects corticosteroid and immunoglobulin concentrations in male house mice (Mus musculus) and prairie voles (Microtus ochrogaster). Comp. Biochem. Physiol. A Physiol. 118: 655663.

33. Shu J, Stevenson JR, Zhou X. (1993) Modulation of cellular immune responses by cold water swim stress in the rat. Dev Comp Immunol 17: 357-371.

34. Song C, Kelly JP, Leonard BE. (1994) The effect of stressful behavioral exposure on endocrine and immune parameters in the rat. Stress Med. 10: 239-245.

35. Nelson RJ. (1997) The use of genetic "knockout" mice in behavioral endocrinology research. Horm. Behav. 31: 188196.

36. Kickhoefer VA, Siva AC, Kedersha NL, et al. (1999) The 193 $\mathrm{kD}$ vault protein, VPARP is a novel poly (ADP-ribose) polymerase. J. Cell Biol. 146: 917-928.

37. Ame JC, Rolli V, Schreiber V, et al. (1999) PARP-2, a novel mammalian DNA damage-dependent Poly (ADP-ribose) polymerase. J. Biol. Chem. 274: 17860-17868. 